\title{
La calcolosi renale. Incomprensioni ed assurdità
}

\author{
N. Di Paolo
}

\author{
Consulente Nefrologo, Azienda Ospedaliera Senese, Siena
}

$\mathbf{L}$ a calcolosi renale in Italia non è certo frequente come nel Nord America, ma esistono sempre cinque probabilità su cento che un italiano possa soffrire nella sua vita di una colica renale e successivamente, in circa il $20 \%$ dei casi, di avere una recidiva. Non è certo poco se si pensa che la calcolosi renale è una malattia che oltre ad essere dolorosa ed invalidante, spesso dà luogo a complicanze: la più frequente di tutte, l'infezione delle vie urinarie, può danneggiare seriamente il rene.

È ben noto che la frequenza della calcolosi, oltre a variare sensibilmente da paese a paese, si presenta in maniera difforme da regione a regione, per poi modificarsi a seconda della familiarità, della professione, dell'alimentazione, dello stato sociale e così via. Tutto ciò da un lato porta a difficoltà negli interventi di prevenzione, dall'altro rende più stimolante la ricerca di fattori favorenti la formazione dei calcoli nelle vie urinarie.

Pur essendo la materia di per sé interessante, nel nostro paese sono pochi i medici o i chirurghi che si dedicano allo studio di questa malattia.

Gli urologi, che più frequentemente vengono a contatto con i pazienti calcolotici, sono spinti, in sintonia con il loro ruolo, ad applicare soluzioni pratiche ed efficienti alla rimozione del calcolo dalle vie urinarie, piuttosto che dedicarsi alla prevenzione della loro formazione e l'avvento della litotrissia extracorporea è, d'altra parte, un successo che premia questo atteggiamento.

Il nefrologo invece, nella maggior parte dei casi si disinteressa della urolitiasi: e quando lo fa si ritrova isolato ad affrontare tematiche difficili e complesse che invece si avvarrebbero di un approccio multidisciplinare e imperniato su studi policentrici.

Ancora più complessa è la situazione della calcolosi urinaria a livello ministeriale. Infatti mentre lo stato assicura all'assistito la litotrissia, omette di informare i pazienti calcolotici e la popolazione a rischio, su come comportarsi in caso di malattia calcolotica renale. In particolare nessuno esorta questi pazienti a frequentare quei pochi Centri specializzati esistenti in Italia dove si effettuano diagnosi cir- costanziate, dove si prescrive un trattamento razionale e dove si attua la prevenzione sia della malattia stessa che delle sue complicanze.

Quanto detto può spiegare, almeno in parte, gli alti numeri di interventi chirurgici effettuati su una buona parte di pazienti calcolotici scarsamente considerati, mal diagnosticati o inadeguatamente trattati. Per capire quanto poco è tenuto in evidenza il problema della calcolosi renale, basta controllare le dimissioni dei pazienti sottoposti a litotrissia, oggi effettuata in un gran numero di nosocomi; nella maggior parte dei casi il paziente viene considerato guarito, compiendo un tragico errore perché è proprio in quel momento che il soggetto ha necessità di essere aiutato per evidenziare, con particolari screening, eventuali errori metabolici e per prevenire le recidive. La prima cosa da attuare, a mio parere, è quella stretta collaborazione fra nefrologo e urologo, sempre tanto predicata, ma solo di rado attuata, se si vuole sperare di agire validamente in questo campo.

L'urologo non può fare a meno di indirizzare il paziente calcolotico al ne- 


\section{Giornale \\ di Tecniche \\ Nefrologiche \\ \& Dialitiche \\ Anno XVI n ${ }^{\circ} 3$ \\ (C) Wichtig Editore 2004}

frologo, una volta rimossi i calcoli dalla via escretrice, onde tentare di attuare un intervento mirato non solo alla prevenzione delle recidive, ma anche ad approntare una razionale terapia medica e dietetica; lo scopo deve essere anche quello di rimuovere eventuali fattori favorenti, e di attuare congiuntamente protocolli razionali di ricerca che permettano di mettere in evidenza la situazione locale rispetto a quella generale.

Il nefrologo, da parte sua, non può logicamente fare a meno dell'urologo in quanto la maggior parte dei calcoli possono essere rimossi solo per via chirurgica o mediante litotrissia. Creare due compartimenti stagni può solo nuocere ai pazienti e contribuire ad aumentare la spesa sanitaria, perché il paziente lasciato a se, ha elevate probabilità di andare incontro a recidive.

Da tutto ciò emerge che un ruolo fondamentale, nel campo della calcolosi renale, spetta al medico di base. Troppo spesso incontriamo pazienti che sapevano da anni di essere affetti da calcolosi renale asintomatica, diagnosticata casualmente durante l'effettuazione di indagini per altri apparati; risulta sempre che non sono stati incoraggiati a compiere indagini specialistiche. Il medico di base è portato di solito a sdrammatizzare il problema fino a che non compare la complicanza.

Indispensabile quindi coinvolgere con campagne informative, tutta la popolazione medica affinché qualsiasi paziente asintomatico che sia portatore di calcoli venga inviato dallo specialista per una valutazione della situazione.

Un problema particolare in cui continuamente mi imbatto, trattando questi malati, spesso affetti anche da infezioni delle vie urinarie, è la difficoltà oggi dì reperire farmaci che hanno ancora una valenza importante in questo campo, ma che purtroppo, in Italia, sono spariti dal commercio. Il farmaco nel nostro paese segue una logica nefanda: a mano a mano che i preparati non risultano più retributivi, vengono ritirati dal commercio, senza che il Ministero della Salute faccia nulla per arginare questo fenomeno prettamente italico dove l'industria propone erroneamente prodotti sosti- tutivi costosissimi, ma in genere non adatti per pazienti che vanno trattati a lungo con farmaci a bassa tossicità. Tanto per fare alcuni esempi ricorderò la sparizione dal mercato italiano (all'estero si trova tutto) della esametilentetramina, dei terpeni, del blu di metilene, dell'elmitolo e di tanti altri preparati che nel campo della calcolosi e dell'infezione delle vie urinarie sono ancora oggi farmaci di indubbia utilità. La cosa più grave, a mio parere, è che la maggior parte di quelli che scrivono di medicina, si adattano all'imposizione del mercato e ignorano nei loro scritti tutti i presidi terapeutici eliminati. È cessata la produzione solo per il difetto di costare poco e non, come qualche volta si vuol fare credere, perché sono stati messi in evidenza effetti indesiderati.

Molto potrebbe essere rimediato con la galenica, ma almeno nel nostro campo, fino ad oggi non si sono visti tentativi di ripristinare medicamenti ancora validi.

In Europa abbiamo anche il problema della terapia idropinica a base di acque minerali: ho volutamente utilizzato il termine "problema" in quanto nel vecchio continente siamo condizionati da millenni di terapia termale i cui risultati sono sotto l'occhio di tutti, ma la dimostrazione scientifica dei quali è ardua e in aperto contrasto con l'ortodossia medica moderna che pone dubbi sulla efficacia della terapia termale, reclamando giustamente la documentazione della validità di un elemento medicamentoso.

Se alcune dimostrazioni sono state fatte, grazie anche ai valenti nefrologi italiani (1) che si occupano di calcolosi, altre ce ne vorranno per chiarire il ruolo della terapia idropinica in questa malattia. A mio parere sono necessari studi seri che i produttori di acque minerali dovrebbero sponsorizzare invece di investire grandi quantità di denaro in una pubblicità che si avvale di messaggi promozionali assolutamente non conformi alla realtà scientifica e che minano la credibilità di questo tipo di terapia.

\section{BIBLIOGRAFIA}

1. Borghi L, Meschi T, Amato F, Briganti A, Novarini A, Giannini A. Urinary volume, water and recurrences in idiopathic calcium nephrolithiasis: a 5-year randomized prospective study, J Urol 1996; 155: 839-43. 